\title{
EXIGENCY OF SELECTING OPTIMAL EXTINCTION RATIO FOR HIGHER SNR AND MINIMAL BER
}

\author{
Tabiya Feroz ${ }^{1}$, Jasiya Bashir ${ }^{2}$, SadafAjaz Khan ${ }^{3}$, Asima Gulzar ${ }^{4}$ \\ ${ }^{I}$ B.E. ECE Undergraduate, Department of Electronics and Communication, University of Kashmir, J\&K, India \\ ${ }^{2}$ B.E. ECE Undergraduate, Department of Electronics and Communication, University of Kashmir, J\&K, India \\ ${ }^{3}$ B.E. ECE Undergraduate, Department of Electronics and Communication, University of Kashmir, J\&K, India \\ ${ }^{4}$ B.E. ECE Undergraduate, Department of Electronics and Communication, University of Kashmir, J\&K, India
}

\begin{abstract}
OFC has made communication faster and safer which has led to its rapid deployment. However, its deployment and reconfiguration is highly uneconomical. Adequate care as such needs to be taken at the time of its deployment so as to ensure minimum possible scope for reconfiguring the system later. For efficient transmission of data, it is to be modulated. In our field of research, we are using Mach Zehnder modulator for modulation purpose. One of the most significant parameter of this modulator is 'Extinction Ratio'. The paper highlights the prominence of this modulation parameter and its effect on the received data.
\end{abstract}

Keywords: BER (Bit Error Rate), Extinction Ratio, Eye Height, Q-factor, SNR.

\section{INTRODUCTION}

In recent years, the optical communication systems have evolved at a rapid pace, therefore Optical communication systems today involve a very high degree of complexity. The design and analysis of these systems, which normally includes multiple signal channels, different topologies, nonlinear devices, noise sources, is highly complex and labor-intensive. Advanced software tools make the design and analysis of these systems quick and efficient [1]. OptiSystem (OptiWave Inc.) is an innovative optical communication system simulation package for the design, testing and optimization of virtually any type of optical link in the physical layer of a broad spectrum of optical networks, from analog video broadcasting systems to intercontinental backbones. OptiSystem represents an optical communication system as an interconnected set of blocks. Each block is simulated independently using the parameters specified by the user for that block and the signal information passed into it from other blocks [3]. As physical signal are passed between components in a real-world communication system, "signal" data is passed between components models in the simulation. These blocks are graphically represented as icons in OptiSystem [4]. Using this system we are capable of changing the parameters individually and then observing the impact of these parameters on our output. The data is transmitted at a particular rate called as data rate. Beyond the data rates of 1$2 \mathrm{GHz}$, the complex dynamics of the coupling of electrons and photons within the laser cavity results in the undesirable frequency variations, known as chirps. In presence of chirp, the signal gets distorted when it propagates through optical fiber, an improved option would be the external modulation. Therefore, for high data rates, bit pattern is modulated by Mach Zehnder Modulator. Within Mach Zehnder, modulation is dependent on a parameter called 'EXTINCTION RATIO'. It is the ratio of two optical power levels engendered by an optical source. The measure of the performance of system is usually expressed in terms of biterror rate (BER), which describes the reliability of the entire radio system from "bits in" to "bits out," including the electronics, antennas and signal path in between. On the surface, BER is a simple concept, its definition is simply:

$$
\text { BER }=\text { Errors } / \text { Total Number of Bits }
$$

For the reimbursement of system degradation, the signal power need to be increased to achieve the same SNR or BER performance as that of an ideal system. This increase in power is called the Power Penalty.

Q-factor symbolizes the quality of a digital signal from an analog point of view, therefore it is referred as a signal to noise ratio. In practical, measurements can be determined as the difference of a signal level from a noise level .For a better reception of information Q-factor (SNR) should be high. Eye diagram is a means of gauging the quality of a received digital waveform. By quality it is meant to correctly recover the symbols and timing. Eye diagram divulge the impact of noise and ISI which are in turn related to BER. The height of the eye gives the margin over noise. The eye height is associated to SNR and thus BER.

Different class of detectors can be used to detect our signal. For our analysis we are using PIN DIODE.

Taking all the above mentioned parameters into considerations we have shown

$>$ The effect of change in extinction ratio of the modulator on the output signal. 


\section{RESEARCH FIELD}

Mostly, whenever data transmission is taken into consideration, output power is the main disquiet. We always want our output power or signal power to be high, but in most of the cases for better output (signal) power ,input power is taken into contemplation .But in our field of research we are scrutinizing the effect of modulator parameter (extinction ratio) on our output power (signal power) and also perceive the effect of this parameter on output signal parameters(Q-factor, min BER, eye height etc).The extinction ratio is one of the most imperative parameter as it gives the measure of the quality of the signal. Also it directly effects the power levels of bits that are being transmitted and also nullifies the effect of noise that is main argument of apprehension on transmitter side.

\section{PROPOSED SYSTEM}

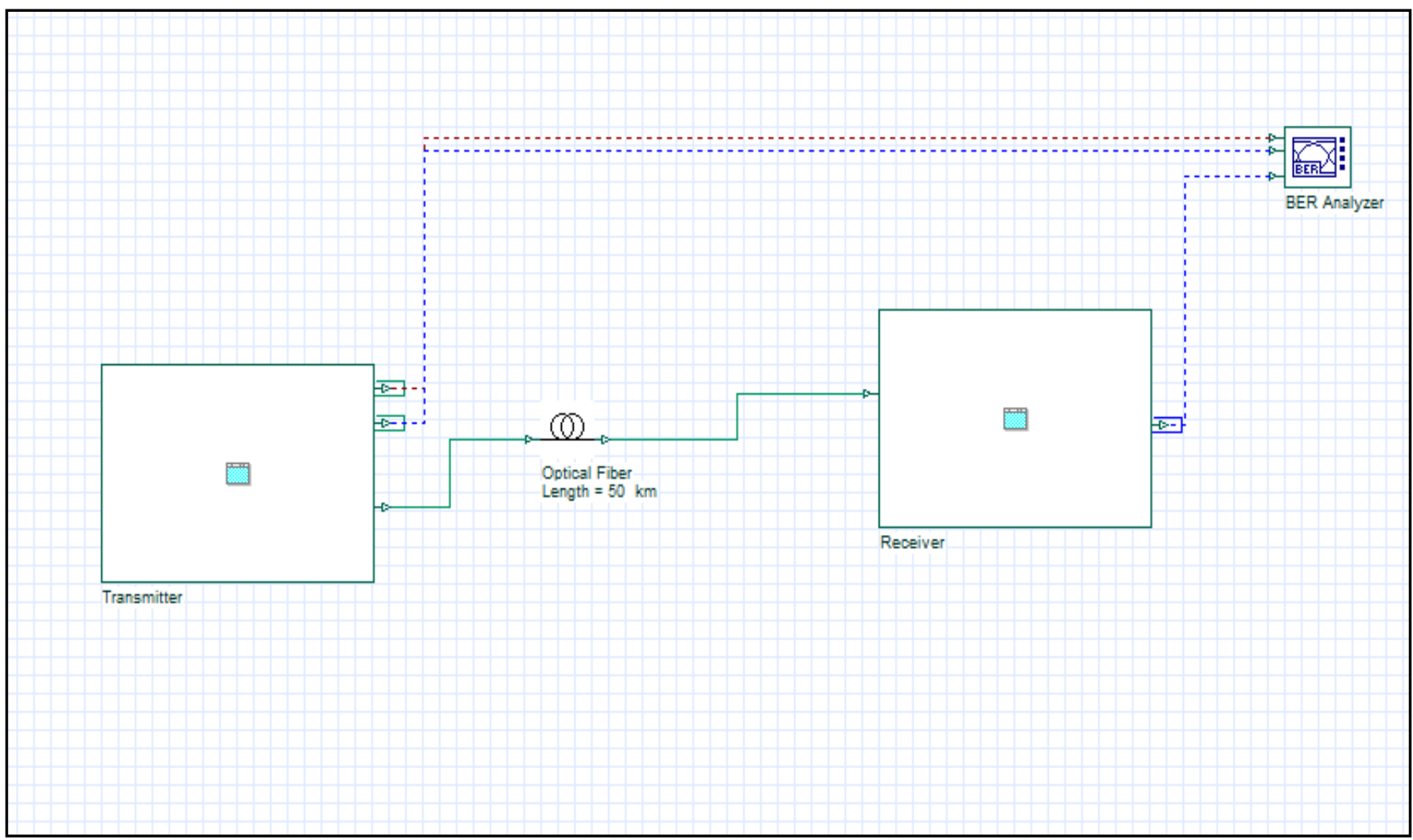

Fig-1: Illustration diagram of the whole system.

\subsection{Transmitter Side}

Transmitter consists of:

i. CW Laser: CW Laser stands for Continuous Wave Laser. The optical sources that we are using in our system are CW Laser. It is set at frequency equal to $193.1 \mathrm{THz}$ and power equal to $0 \mathrm{dBm}(1 \mathrm{~mW})$. For frequencies that are greater than $1-2 \mathrm{GHz}$, modulator is used. The modulator that we are using for such frequencies is Mach Zehnder modulator.

ii. MachZehnder modulator: As the demand for high speed Communication is increasing, the need for external modulation in Optical transmission systems is also increasing. One of the possibilities of external modulation is to practise a Mach-Zehnder structure in a material showing strong electro-optic effect (such as LiNbO3). In Mach Zehnder, voltage is applied to both the arms causing a change in their refractive index according to electro-optic effect. This electro-optic effect is caused due to the application of electrical signal from NRZ Pulse generator and optical signal from CW Laser. This changing refractive index phase modulates the beam propagating through arms of interferometer according to applied voltage. This phase modulation gets converted to intensity modulation by combining the two paths [2]. 


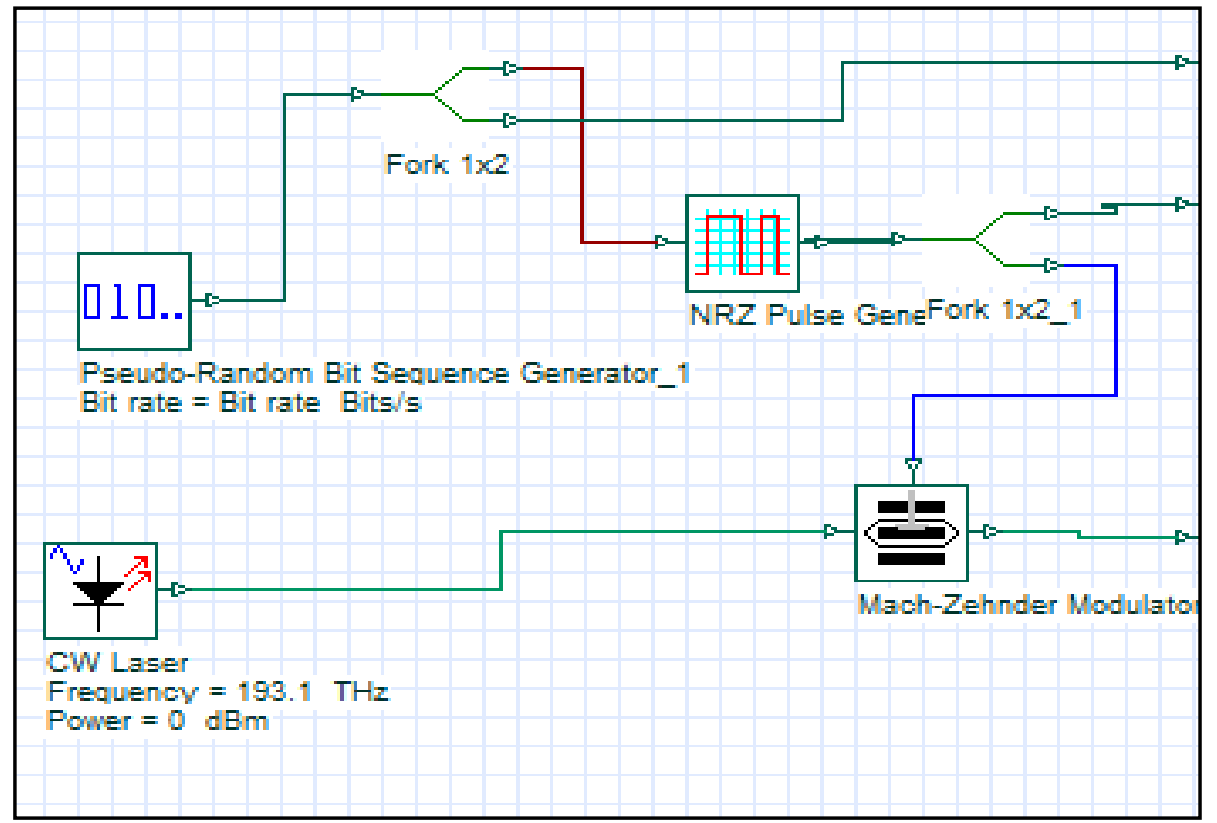

Fig-2: Illustration diagram of transmitter side.

iii. NRZ Pulse generator: NRZ pulse generator crafts a sequence of non-return to zero pulses coded by an input digital signal. This digital signal is provided by Pseudo- random Bit Sequence generator. It has been found that the NRZ generator are mostly used for all simulation results.

\subsection{Receiver Side}

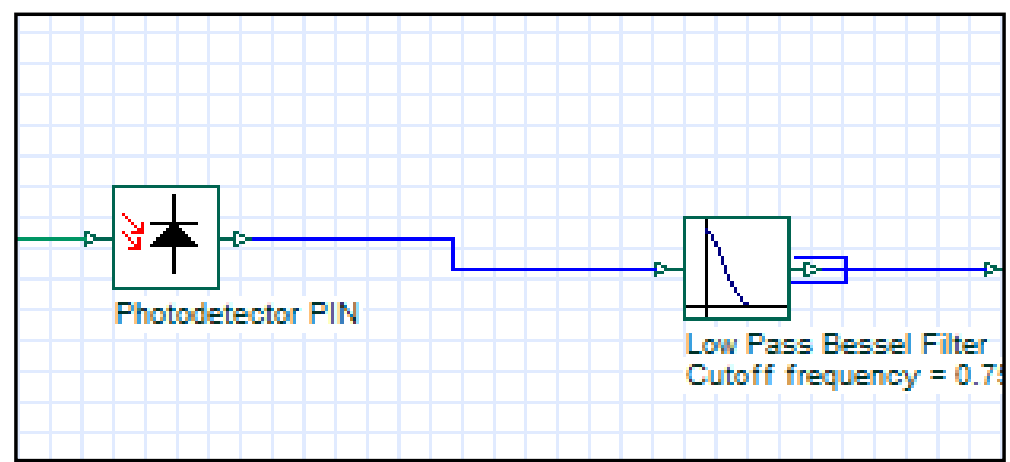

Fig-3 Illustration diagram of the receiver side.

Receiver side consists of:

i. Photodetector PIN: PIN photodiode is a kind of photodetector, it converts optical signals into electrical signals. It is the most noteworthy part of receiver portion. But the limitation of this photodiode is that it is not precise i.e., along with the desired information it also incorporates noise with the required information, as a result of which unsolicited frequencies are also detected along with the required frequency. So to circumvent this we make use of a filter.

ii. Low Pass Bessel Filter: It is a type of analog linear filter, which conserves the shape of the filtered signals in pass band.

\subsection{Transmission Medium}

It is used to convey the information from transmitter side to receiver side.

\subsection{Analysers}

They are used to perceive the change in the concerned parameters (Q-factor, min BER, Eye height, Threshold) caused due to our proposed system. The analyser that we are using here is BER Analyser. It entails two electrical and one optical input. These inputs are acquired from pseudorandom bit sequence generator, NRZ pulse generator and low pass Bessel filter respectively. 


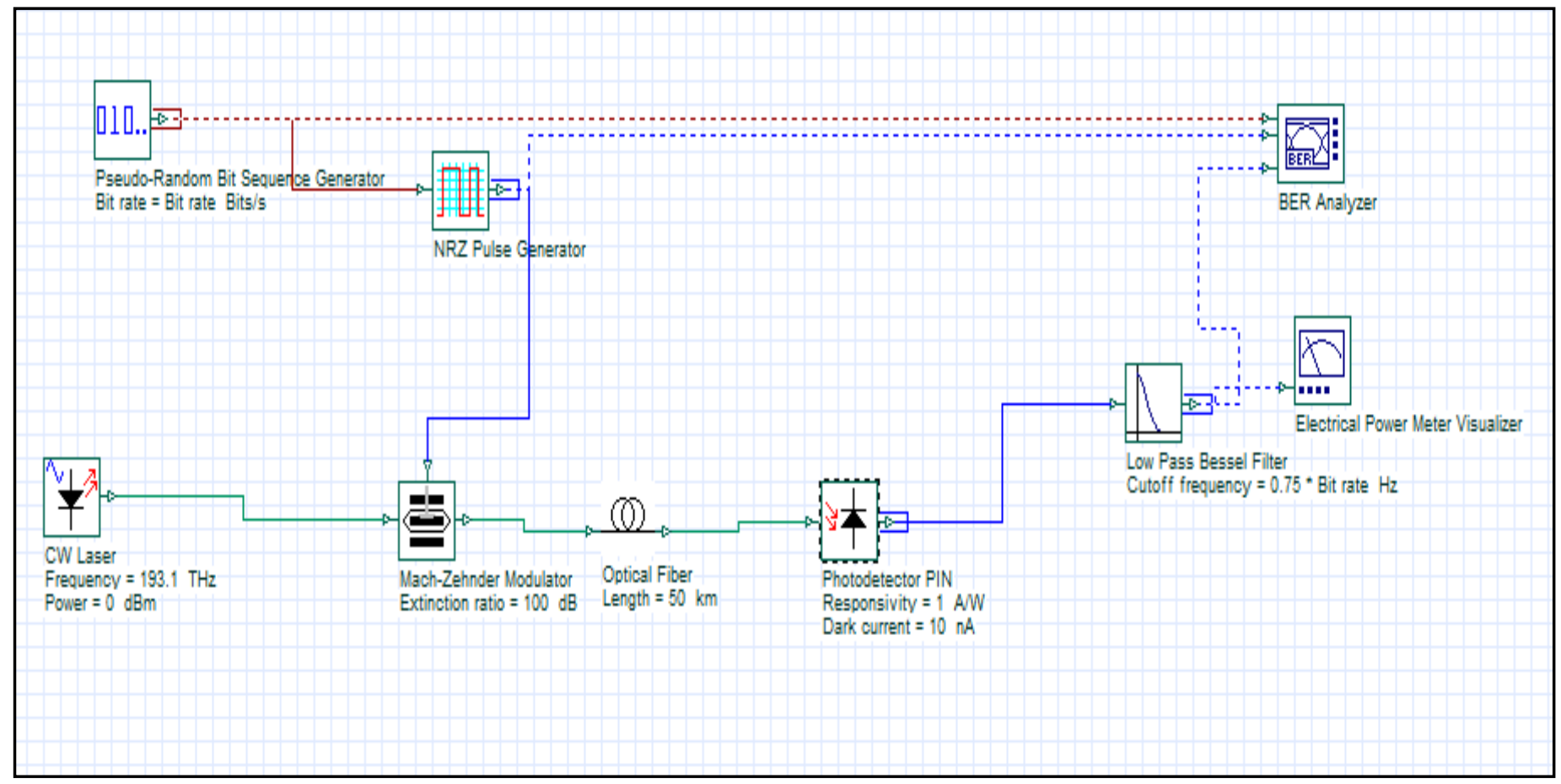

Fig-4: Illustration diagram of BER analyser

\section{RESULTS AND GRAPHS}

Effect of extinction ratio on the signal power: In general, with the increase in extinction ratio, signal power increases. This can be easily shown by the graph (fig 5).

It is quite clear from the fig.5 that on increasing extinction ratio our signal power is increasing. This is due to the fact that we are actually increasing the power levels of 1's and 0 's that are being transmitted.

Table-1:Variation of signal power with respect to extinction ratio

\begin{tabular}{|cl|}
\hline Extinction & Signal power in \\
ratio $\left(\mathrm{r}_{\mathrm{e}} \mathrm{in \textrm {dB }}\right)$ & $\mathrm{E}-9(\mathrm{~W})$ \\
10 & 3.707 \\
20 & 4.286 \\
30 & 4.373 \\
40 & 4.388 \\
50 & 4.391 \\
60 & 4.392 \\
70 & 4.393 \\
80 & 4.393 \\
90 & 4.393 \\
100 & 4.393 \\
\hline
\end{tabular}

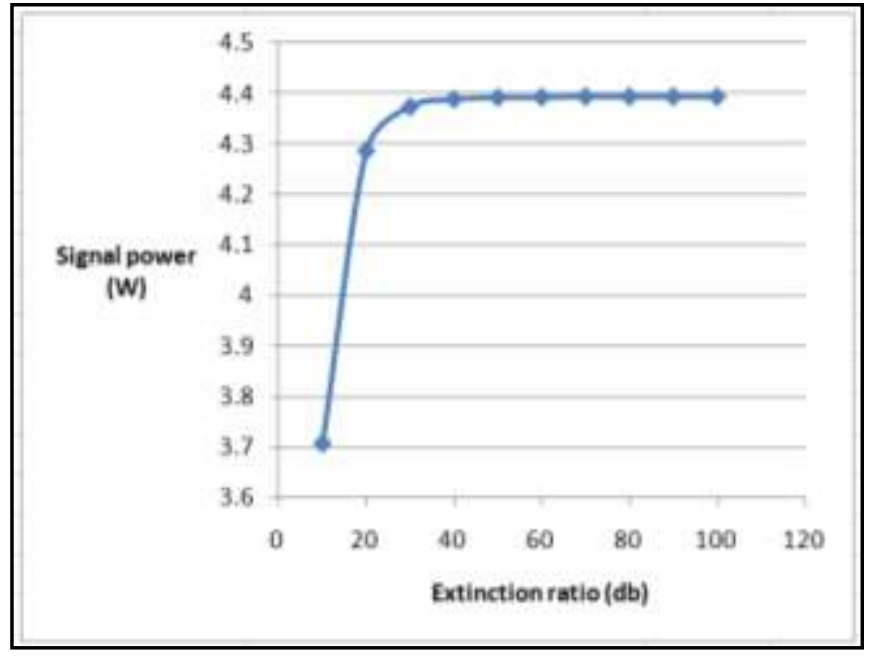

Fig-5: Graphical illustration showing effect of extinction ratio on signal power

Table: 2 Variation of SNR with respect to extinction ratio

\begin{tabular}{|cl|}
\hline $\begin{array}{c}\text { Extinction } \\
\text { Ratio }\left(\mathrm{r}_{\mathrm{e}} \text { in } \mathrm{dB}\right)\end{array}$ & $\begin{array}{c}\text { Signal to noise } \\
\text { ratio }(\mathrm{SNR})\end{array}$ \\
10 & 2.246666667 \\
20 & 2.615009152 \\
30 & 2.672982885 \\
40 & 2.682151589 \\
50 & 2.68398533 \\
60 & 2.684596577 \\
70 & 2.686850153 \\
80 & 2.686850153 \\
90 & 2.686850153 \\
100 & 2.686850153 \\
\hline
\end{tabular}




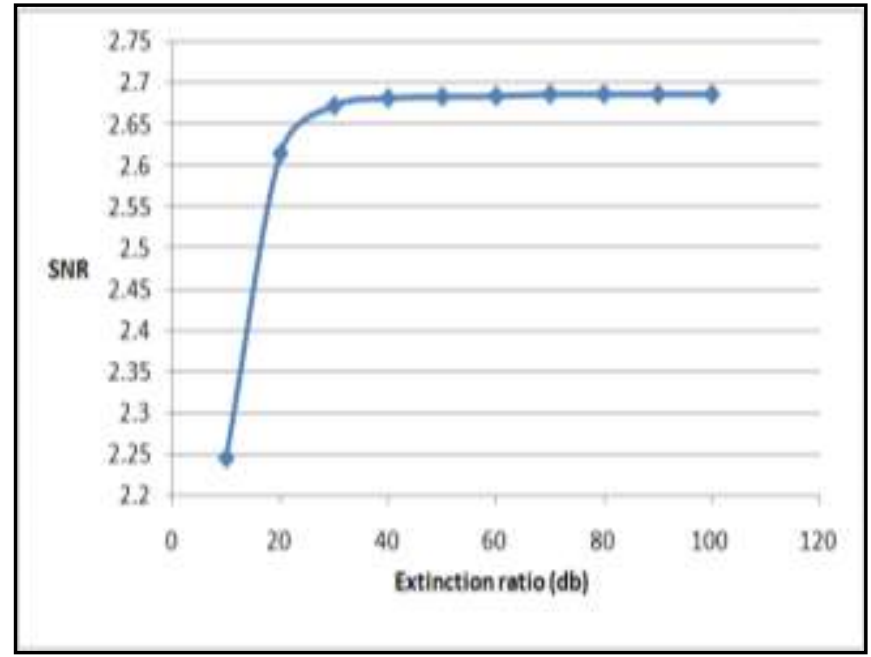

Fig-6: Graphical illustration showing effect of extinction ratio on $\mathrm{SNR}$

From the fig. 6 it is reasonably clear that on increasing extinction ratio (means we are increasing the power levels of bits that are being transmitted), SNR is increasing as expected.

Table-3: Variation of eye height with respect to extinction ratio

\begin{tabular}{|ll|}
\hline Extinction & Eye height \\
Ratio $\left(\mathrm{r}_{\mathrm{e}}\right.$ in $\left.\mathrm{dB}\right)$ & $($ in $\mu \mathrm{m})$ \\
10 & 6.332 \\
20 & 7.135 \\
30 & 7.025 \\
40 & 6.952 \\
50 & 6.92513 \\
60 & 6.9162 \\
70 & 6.91333 \\
80 & 6.91242 \\
90 & 6.91213 \\
100 & 6.91204 \\
\hline
\end{tabular}

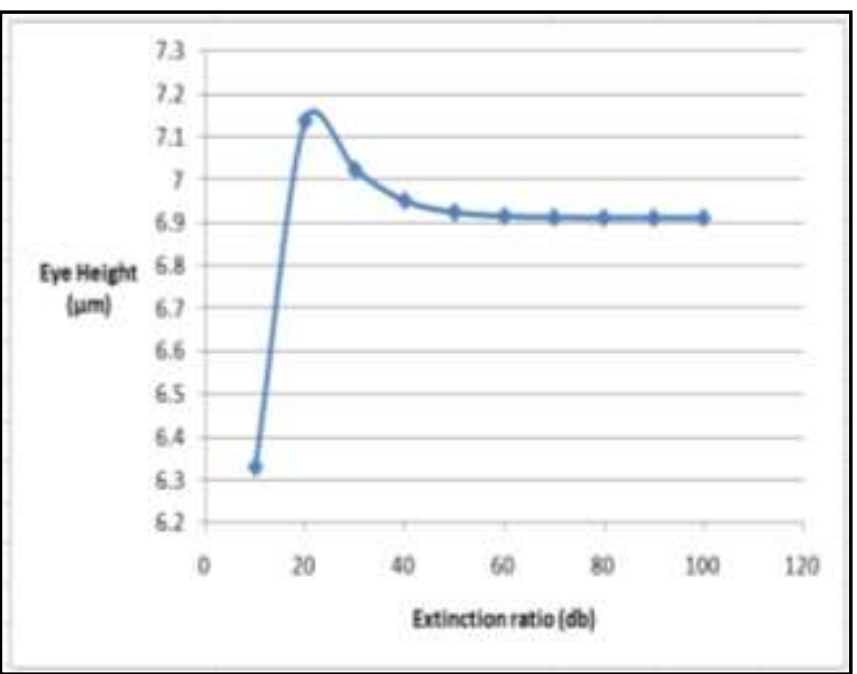

Fig-7: Graphical illustration showing effect of extinction ratio on eye height.

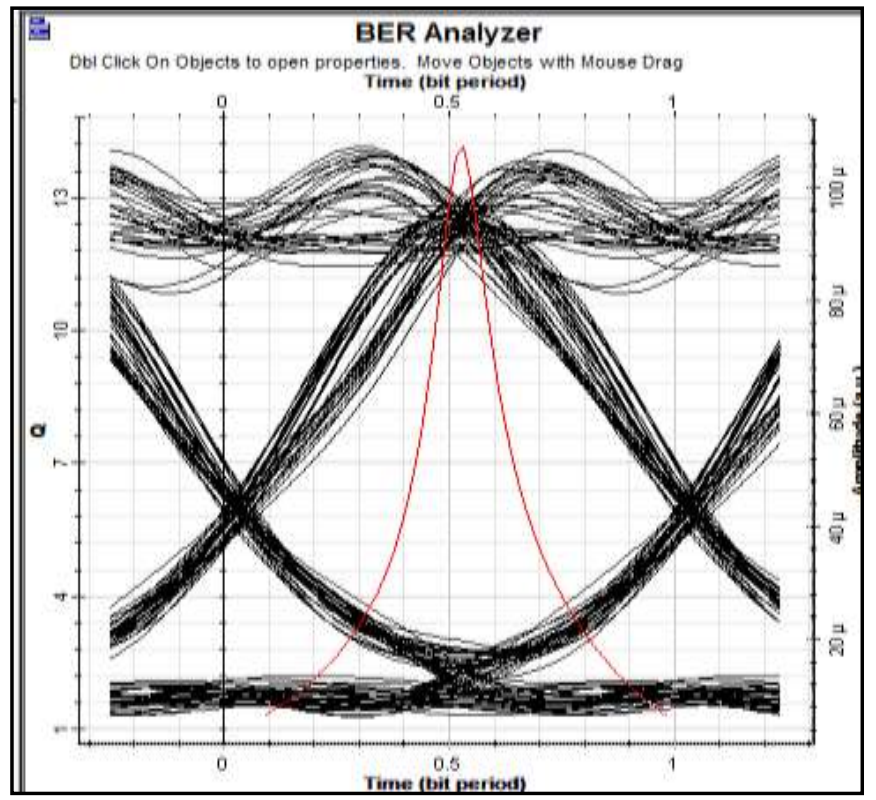

Fig-8: Eye diagram at extinction ratio $r_{e}=10 \mathrm{db}$

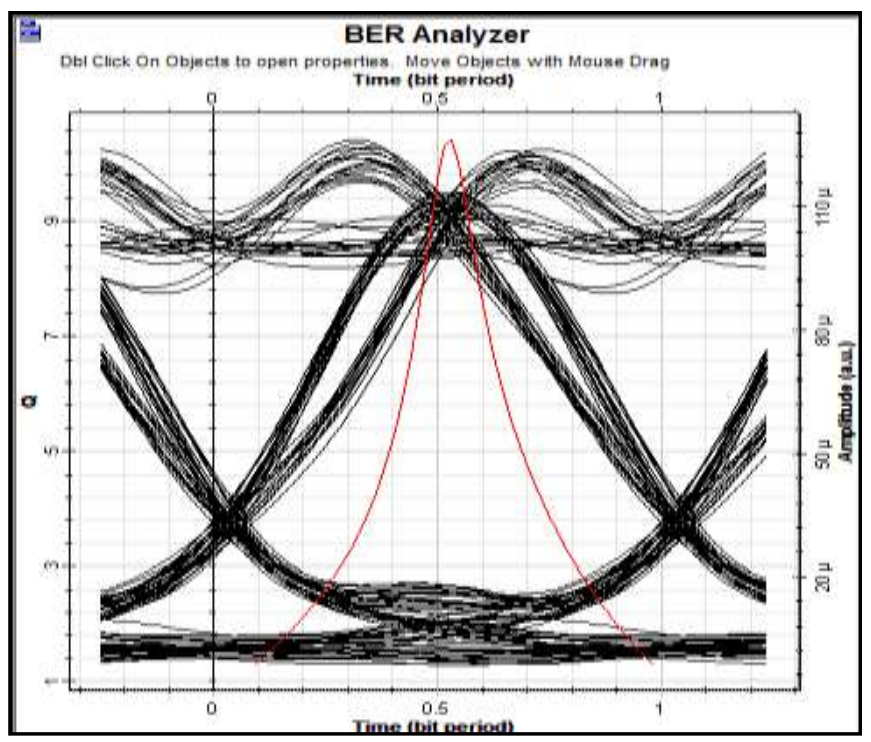

Fig-9: Eye diagram at extinction ratio $r_{e}=100 \mathrm{db}$

Table-4: Variation of threshold with respect to Extinction ratio

\begin{tabular}{|ll|}
\hline Extinction & Threshold \\
Ratio $\left(\mathrm{r}_{\mathrm{e}}\right.$ in $\left.\mathrm{dB}\right)$ & \\
10 & 5.69847 \\
20 & 6.2075 \\
30 & 6.26822 \\
40 & 6.2789 \\
50 & 6.28156 \\
60 & 6.28233 \\
70 & 6.28256 \\
80 & 6.28264 \\
90 & 6.28266 \\
100 & 6.28267 \\
\hline
\end{tabular}




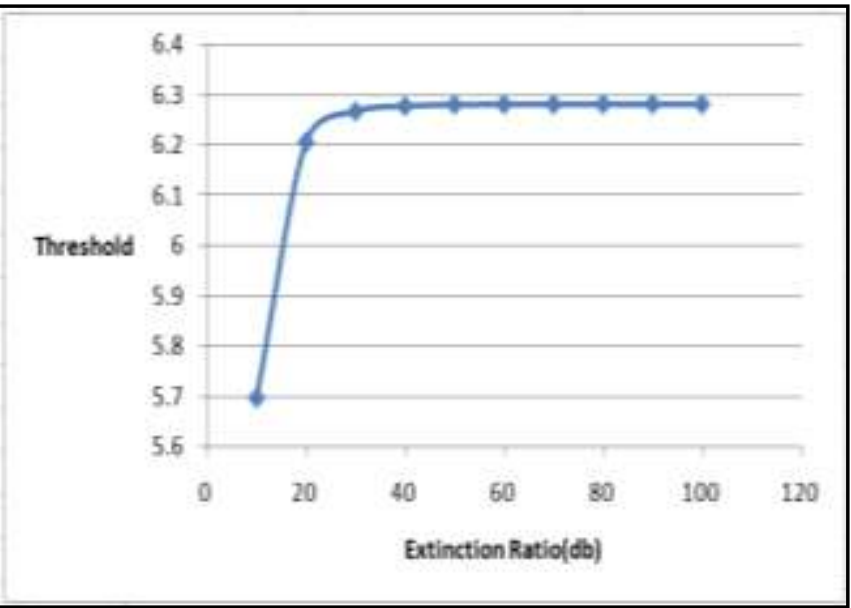

Fig-10: Graphical illustration showing effect of extinction ratio on threshold

Table-5: Variation of BER with respect to extinction ratio

\begin{tabular}{|cc|}
\hline Extinction & Bit error \\
Ratio $\left(r_{\mathrm{e}}\right.$ in $\left.\mathrm{dB}\right)$ & rate $(\mathrm{BER})$ \\
10 & $1.67 \mathrm{E}-47$ \\
20 & $1.77 \mathrm{E}-31$ \\
30 & $2.49 \mathrm{E}-26$ \\
40 & $8.06 \mathrm{E}-25$ \\
50 & $2.35 \mathrm{E}-24$ \\
60 & $2.39 \mathrm{E}-24$ \\
70 & $3.66 \mathrm{E}-24$ \\
80 & $3.78 \mathrm{E}-24$ \\
90 & $3.82 \mathrm{E}-24$ \\
100 & $3.84 \mathrm{e}-24$ \\
\hline
\end{tabular}

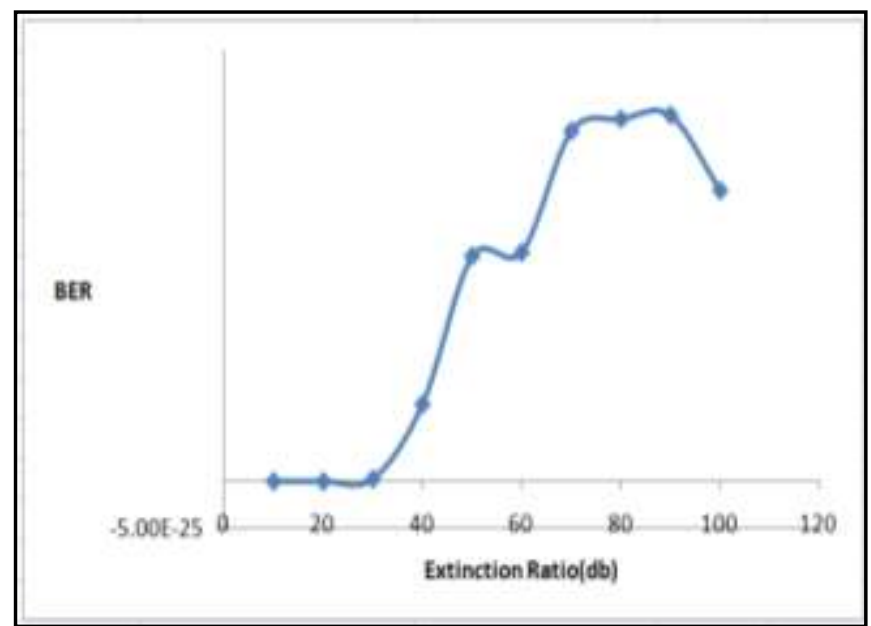

Fig-11: Graphical illustration showing effect of extinction ratio on BER

From the fig. 10 it is vibrant that with the increase in the extinction ratio our threshold increases. This means that the threshold required for depicting the change in symbols increases, thus making it difficult for the receiver to detect the symbol with accuracy. Hence, this in turn increases BER (as shown in fig.11).

\section{CONCLUSION}

Extinction ratio is a key parameter for noise free transmission as well as for error free reception of data. From our analysis, we endowed that with increase in Extinction Ratio, Signal to Noise power (SNR) increases that aids in better reception of signal. Moreover with increase in Extinction ratio, BER increases as threshold that separates the bit levels increases, making it difficult for the receiver to detect the original data. So, we accomplish that for better transmission and reception of data, we require to select the optimum value of extinction ratio, neither too high nor too low.

\section{REFERENCES}

[1] http://www.optiwave.co.kr/product/brochures.htm

[2] K P Zetie, S F Adams and R M Tocknell Physics Department, Westminster School, London SW1 3PB, UK

[3] Opt wave__opt system "Optiperformer version7.0UserGuide”, Optical communication system software.

[4] Xiang Yang, Yang Hechao,'The Application of Optisystem in Optical Fiber Communication Experiments", Proceedings of the Third International Symposium on Computer Science and Computational Technology(ISCSCT'10),pp.376-378,August 2010. 